\title{
STUDYING AURORAL ACTIVITY USING THE SME INDEX AT THE MAGNETIC STORM MAIN PHASE DURING CIR AND ICME EVENTS
}

\author{
R.N. Boroyev \\ Yu.G. Shafer Institute of Cosmophysical Research \\ and Aeronomy of SB RAS, FRC YaSC SB RAS, \\ Yakutsk, Russia,boroyev@ikfia.ysn.ru \\ M.K. Ammosov North-Eastern Federal University, \\ Yakutsk, Russia
}

\author{
M.S. Vasiliev \\ Yu.G. Shafer Institute of Cosmophysical Research \\ and Aeronomy of SB RAS, FRC YaSC SB RAS, \\ Yakutsk, Russia,ms_vasiliev@ikfia.ysn.ru \\ M.K. Ammosov North-Eastern Federal University, \\ Yakutsk, Russia
}

\begin{abstract}
In this paper, we examine the relationship of the $S M E$ index with magnetic storm characteristics and interplanetary medium parameters during the main phase of magnetic storms caused by CIR and ICME events. Over the period 1990-2017, 107 magnetic storms driven by (64) CIR and (43) ICME events have been selected. In contrast to $A E$ and $K_{\mathrm{p}}$, a stronger correlation is shown to exist between the average $S M E$ index $\left(S M E_{\text {aver }}\right)$ and interplanetary medium parameters during the magnetic storm main phase. Close correlation coef-
\end{abstract}

ficients between $S M E_{\text {aver }}$ and the SW electric field (southward IMF $B_{z}$ ) have been obtained for CIR and ICME events. $S M E_{\text {aver }}$ has been found to increase with the rate of magnetic storm development and $\left|D s t_{\min }\right|$. For CIR and ICME events, no difference has been revealed between $S M E_{\text {aver }}$ and $\left|D s t_{\min }\right|$ in linear regression equations.

Keywords: magnetic storm, $S M E$ index, Dst index, solar wind, electric field.

\section{INTRODUCTION}

It is known that amplification of magnetosphericionospheric currents during magnetic storms leads to an increase in the geomagnetic activity indices $D s t, A E, K_{\mathrm{p}}$. The geomagnetic indices, calculated from ground-based observations, describe the dynamics and intensity of magnetic disturbances during magnetic storms. The lowlatitude Dst index [Sugiura, 1964; Burton et al., 1975] is used to estimate the magnetic storm intensity. The highlatitude $A E$ index characterizes the intensity of the auroral current and is an indicator of substorm activity [Davis, Sugiura, 1966]. Auroral activity is also assessed by the planetary (mid-latitude) $K_{\mathrm{p}}$ index [Bartels, 1949; Khorosheva, 2007]. Correlation analysis of geomagnetic activity and interplanetary medium parameters during magnetic storms has shown that the southward interplanetary magnetic field component $B_{z}$, whose efficiency is associated with the effect of the SW electric field $E_{\mathrm{sw}}=V \times B_{z}$, is the main driver of geomagnetic disturbances [Burton et al., 1975; Gonzalez et al., 1994; Kane, 2005, 2010]. The relationship between $A E, K_{\mathrm{p}}$, and Dst indices during magnetic storms has been extensively discussed in [Gonzalez et al., 1994; Grafe et al., 1997; Grafe, Feldstein, 2000; Kane, 2010] (see also references therein).

However, the results of recent statistical and morphological studies suggest that the development of magnetic storms and substorms differs depending on the type of the solar wind (SW) [Gonzalez et al., 1999; Borovsky, Denton, 2006; Yermolaev et al., 2010; Dremukhina et al., 2018a]. The following SW types are distinguished: interplanetary coronal mass ejections (ICME) including magnetic clouds (MC) and pistons (ejecta), regions of interaction between fast and slow streams (CIR), and compression regions before ICME (sheath). Each SW type has a specific set of SW and IMF parameters.

Analysis of the relationship between SW parameters for different types of streams and the geomagnetic activity indices $\left(A E, K_{\mathrm{p}}\right.$, and $\left.D s t\right)$ has revealed [Plotnikov, Barkova, 2007; Yermolaev et al., 2010; Guo et al., 2011; Nikolaeva et al., 2011; Yermolaev et al., 2012; Cramer et al., 2013; Dremukhina et al., 2018a] that during storm main phases the geomagnetic indices increase with increasing SW electric field $E_{\mathrm{sw}}$, but for MC events there is a nonlinear dependence of the indices on $E_{\mathrm{sw}}$. Boroyev et al. [2020] have carried out a comparative analysis of the $A E$ and $K_{\mathrm{p}}$ indices during CIR and ICME events. It has been found that for CIR events, in contrast to ICME (MC + ejecta), $A E$ increases with $E_{\mathrm{sw}}$. The $K_{\mathrm{p}}$ index correlates with $E_{\mathrm{sw}}$ only for ICME. The authors of [Borovsky, Denton, 2006; Plotnikov, Barkova, 2007; Yermolaev et al., 2010] attribute the observed variations in the auroral indices to SW stream type. Differences between SW types are manifested in the behavior of the ring current, auroral precipitation, Earth's plasma sheet, magnetospheric convection, and saturation of the polar cap potential [Borovsky, Denton, 2006]. In [Boroyev, Vasiliev, 2018; Boroyev et al., 2020], the authors believe that variations in the $A E$ and $K_{\mathrm{p}}$ indices are influenced by the position of the auroral current relative to the stations at which the indices are calculated. A significant decrease in IMF $B_{z}$ leads to an expansion of the auroral oval [Akasofu, Chapman, 1974] and to the drift of ionospheric currents to lower latitudes. Boroyev et al. [2020] assume that it is the SW type that determines the drift of currents relative to the stations at which $A E$ and 
$K_{\mathrm{p}}$ are calculated. For example, during ICME events, unlike CIR events, large values of the southward IMF $B_{z}$ are observed. As a result, we can see higher correlation coefficients between $E_{\text {swaver }}$ and $K_{\text {paver }}$ than between $E_{\text {swaver }}$ and $A E_{\text {aver }}$ during the main phases of ICMEinduced magnetic storms.

To take into account the spatial features of the influence of the equatorial drift of the auroral electrojet on $A E$ and $K_{\mathrm{p}}$ during magnetic storms, we examine the SuperMAG SME index. The SuperMAG indices $S M E, S M U$, and $S M L$ are geomagnetic activity indices [Newell, Gjerloev, 2012]. Calculation formulas, say, for $A E$ and $S M E$, are similar. The $A E$ index is calculated from the $H$ component at twelve high-latitude magnetovariational stations located in a narrow band of geomagnetic latitudes $65^{\circ}$ $70^{\circ}$. The $S M E$ index is calculated using data from more than 100 geomagnetic stations [Newell, Gjerloev, 2011], covering the range of geomagnetic latitudes from $40^{\circ}$ to $80^{\circ}$. The $S M E$ index, unlike $A E$, allows for a more accurate assessment of the auroral electrojet intensity. Besides, during magnetic disturbances the shift of the auroral electrojet in latitude does not affect the $S M E$ index. Unlike $S M E, A E$ values are underestimated due to the shift of the auroral electrojet to low latitudes for magnetic storms with $D s t<-60 \mathrm{nT}$ [Khorosheva, 2007]. Using the SuperMAG database, Newell et al. [2013] have examined annual, seasonal, and diurnal variations in the rate of occurrence of substorm disturbances in 1984-2005. Du et al. [2018] have analyzed semiannual and annual variations in $S M L / S M U$, taking into account $\mathrm{SW}$ parameters.

The purpose of this work is to continue the cycle of studies of auroral activity during magnetic storms induced by SW of different types, namely, to examine the correlations of the $S M E$ index with magnetic storm characteristics and interplanetary medium parameters during main phases of magnetic storms induced by SW of different types.

\section{EXPERIMENTAL DATA}

To assess geomagnetic activity, we have used the SME and Dst indices. Their values were taken on the websites [https://supermag. jhuapl.edu, http://wdc.kugi.kyotou.ac.jp/dstae/index.html]. For the period 1990-2017, we have selected 107 magnetic storms with $D s t_{\min } \leq-50 \mathrm{nT}$, induced by CIR (64) and ICME (43) events. We do not deal with other SW types here. A magnetic storm is considered driven by SW of a given type if the main phase and the minimum Dst index were observed under the impact of SW of this type. A classification method for different SW types is described in detail in [Yermolaev et al., 2009; Yermolaev et al., 2010]. The website [ftp.iki.rssi.ru/pub/ omni/catalog] contains a catalog of SW types. Of the ICME events before which a sheath was detected we have selected only those magnetic storms for which the main phase and minimum Dst were observed during ICME (ICME body). It is known (e.g., [Yermolaev et al., 2021]) that the efficiency in magnetic storm generation by sheath is $50 \%$ higher than that by ICME. Therefore, we analyze events occurring after a magnetically quiet period, which allow us to more accurately determine whether the magnetosphere interacts with ICME or sheath during magnetic storm main phases.

For each event, as in [Boroyev, Vasiliev, 2018], we calculate mean $S M E$ values during the magnetic storm main phase and the rate of magnetic storm development as the time derivative of $D s t|\Delta D s t| / \Delta T$ [Ermolaev et al., 2015]. The duration of the main phase $\Delta T$ is defined as the time interval from the moment of a sharp decrease in $D s t\left(D s t_{0}\right)$ to the moment of detection of $D s t_{\min }|\Delta D s t|$ is calculated by the formula

$$
|\Delta D s t|=\left|D s t_{\min }-D s t_{0}\right| \text {. }
$$

To take into account the SW and IMF parameters, we use hourly average data [http://www.omniweb.com] to find mean values of the SW azimuthal electric field $E_{\text {swaver }}=\Sigma E_{\text {sw }} / \Delta T$ and IMF $B_{z} B_{\text {zaver }}=\Sigma B_{\mathrm{z}} / \Delta T$ during the magnetic storm main phase, where $\Sigma E_{\mathrm{sw}}$ and $\Sigma B_{z}$ are the total values of the dawn-dusk electric field and southward $B_{z}$ for the time interval corresponding to the magnetic storm main phase. The average values of the interplanetary medium parameters and the geomagnetic activity indices allow us in general to assess the development of the magnetic storm main phase.

We have used a linear approximation as the simplest way to establish the relationship of the SME index with the magnetic storm and interplanetary medium parameters. In this work, linear approximations and Pearson correlation coefficients are calculated with a probability $P=0.99$ and a significance level $p=0.01$.

\section{RESULTS}

Figure 1, $a, c$ illustrates a correlation between average $S M E S M E_{\text {aver }}$ and the rate of magnetic storm development $|\Delta D s t| / \Delta T$ during CIR and ICME events. The relationship between $S M E_{\text {aver }}$ and $\left|D s t_{\min }\right|$ is displayed in Figure $1, b, d$. Table lists correlation coefficients between $S M E_{\text {aver }}$ and magnetic storm $|\Delta D s t| / \Delta T,\left|D s t_{\min }\right|$ and interplanetary medium parameters, as well as linear regression equations, for $\mathrm{SW}$ of two types. Figure 1, $a, c$ shows that in magnetic storm main phases during CIR

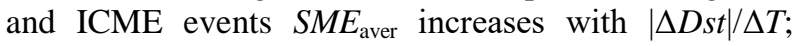
$S M E_{\text {aver }}$ increasing more sharply for ICME events (see Table). We have obtained close correlation coefficients between $S M E_{\text {aver }}$ and $|\Delta D s t| / \Delta T$ for CIR $(r=0.5)$ and ICME $(r=0.54)$ events. For CIR $(r=0.67)$ and ICME $(r=0.6)$ events, high correlation coefficients are also observed between $S M E_{\text {aver }}$ and $\left|D s t_{\min }\right|$ (Figure $1, b, d$ ). Regression equations for $S M E_{\text {aver }}$ and $\left|D s t_{\min }\right|$ for CIR and ICME events are similar (see Table): the angular coefficients that determine the slope of straight lines relative to the $\mathrm{X}$-axis differ slightly.

Among the IMF and SW parameters, the electric field is the main driver of magnetic storms [Burton et al., 1975; Gonzalez et al., 1994; Kane, 2005]. The SW electric field is related to the SW plasma velocity $V_{\text {sw }}$ and one of the significant geoeffective interplanetary medium parameters - the southward IMF $B_{z}$ component. In terms of SW type, we have performed a correlation analysis of $S M E_{\text {aver }}$, the SW electric field, and the southward $B_{z}$ component.

Figure 2 exhibits the correlation between $S M E_{\text {aver }}$ and $E_{\text {swaver }}$ and $\left|B_{z \text { aver }}\right|$ for CIR- and ICME-induced magnetic storms. Figure 2 indicates that for SW of both 

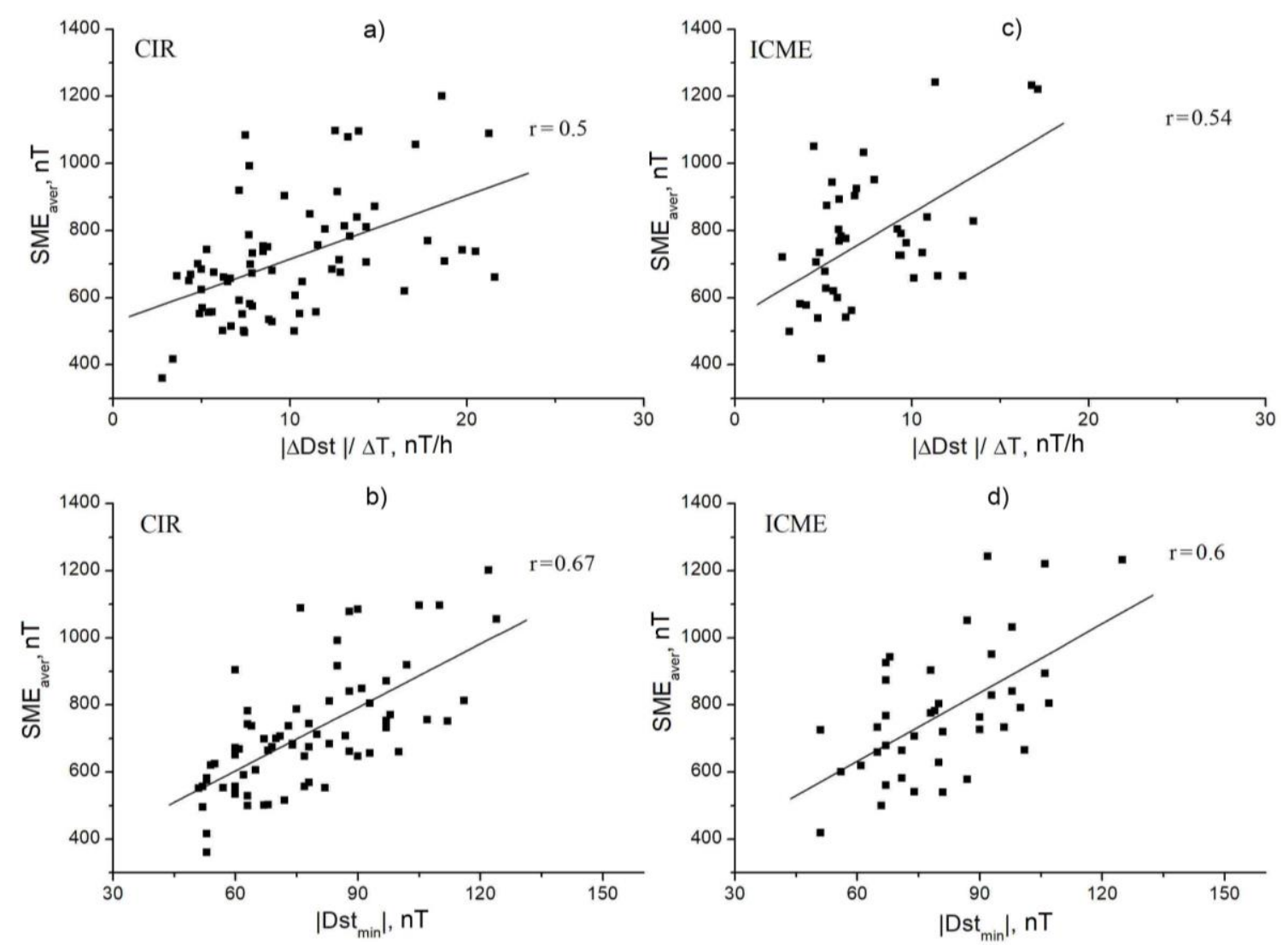

Figure 1. $S M E_{\text {aver }}$ as a function of the magnetic storm development rate and the $D s t_{\min }$ modulus during main phases of CIR- and ICME-induced magnetic storms: squares — separate magnetic storms; straight lines — linear approximation; $r$ correlation coefficient

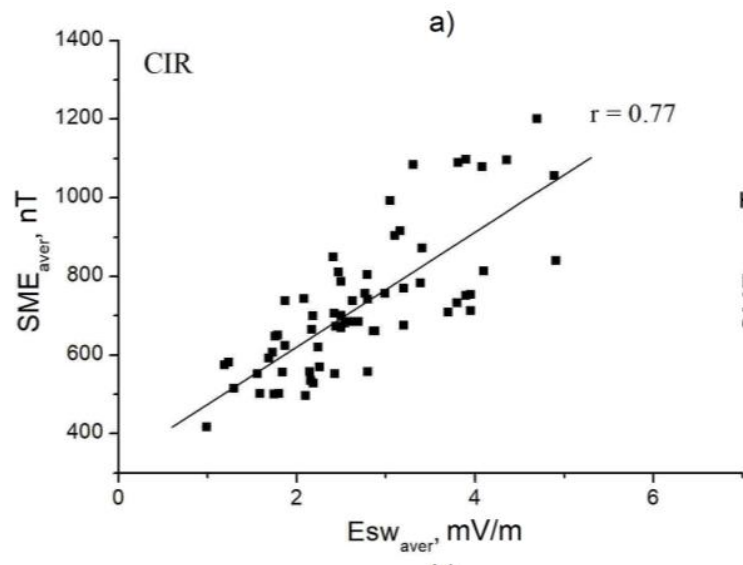

b)

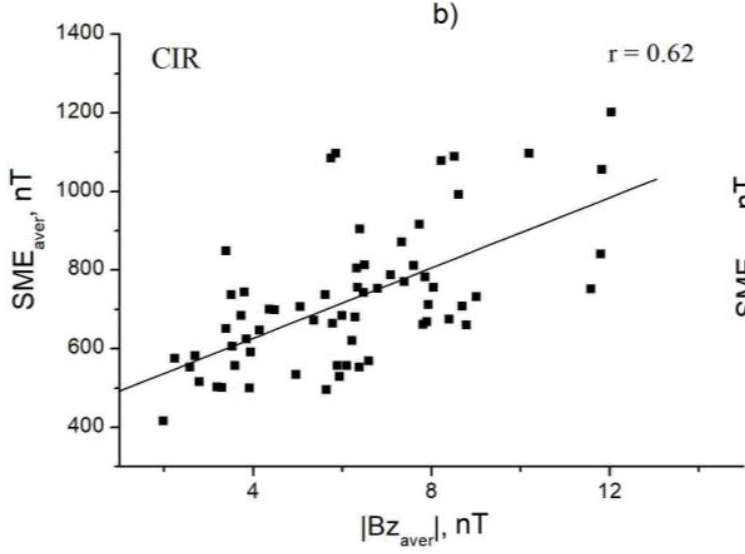

c)

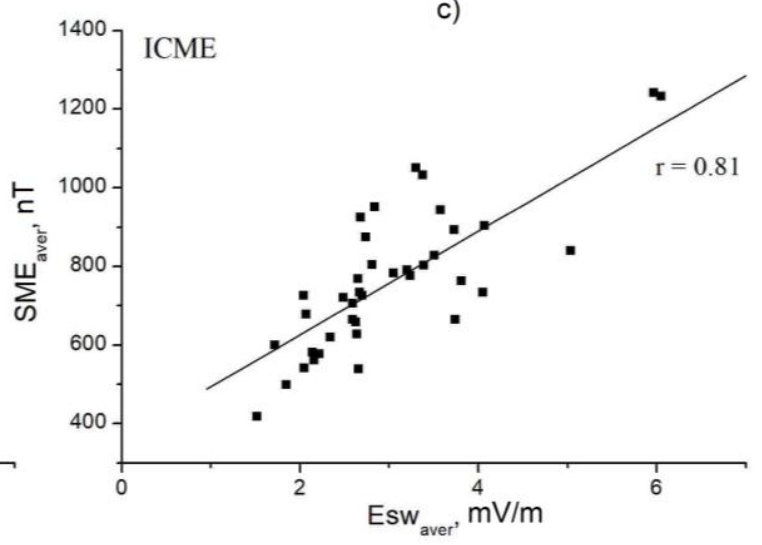

d)

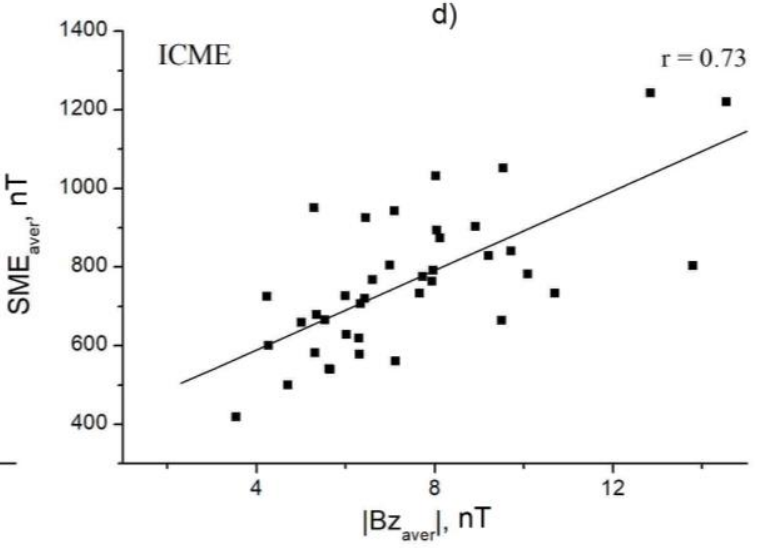

Figure 2. $S M E_{\text {aver }}$ versus the mean values of the SW electric field and the southward IMF $B_{z}$ modulus during main phases of CIR- and ICME-induced magnetic storms 
Pearson correlation coefficients $r$ between $S M E_{\text {aver, }}$, magnetic storm characteristics, and interplanetary medium parameters, calculated with a significance level $p=0.01$, in magnetic storm main phases during CIR and ICME events, as well as regression equations. The correlation coefficients and regression equations for $A E$ and $K_{\mathrm{p}}$ have been taken from [Boroyev et al., 2020]

\begin{tabular}{|l|c|c|l|l|}
\hline & CIR & ICME & \multicolumn{2}{|c|}{$\begin{array}{c}\text { Regression equations for } \\
S M E_{\text {aver }}\end{array}$} \\
\cline { 2 - 5 } & $r$ & $r$ & \multicolumn{1}{|c|}{ CIR } & \multicolumn{1}{c|}{ ICME } \\
\hline$S M E_{\text {aver }}$ and $|\Delta D s t| / \Delta T$ & 0.50 & 0.54 & $y=20 x+526$ & $y=29 x+551$ \\
\hline$A E_{\text {aver }}$ and $|\Delta D s t| / \Delta T$ & 0.51 & 0.29 & $y=14 x+485$ & $y=9.7 x+572$ \\
\hline$K_{\text {aver }}$ and $|\Delta D s t| / \Delta T$ & 0.54 & 0.62 & $y=0.8 x+40$ & $y=1.2 x+34$ \\
\hline$S M E_{\text {aver }}$ and $\left|D s t_{\min }\right|$ & 0.67 & 0.6 & $y=6.3 x+226$ & $y=6.8 x+223$ \\
\hline$A E_{\text {aver }}$ and $\left|D s t_{\min }\right|$ & 0.53 & 0.41 & $y=4 x+320$ & $y=2 x+464$ \\
\hline$K_{\text {paver }}$ and $\left|D s t_{\min }\right|$ & 0.59 & 0.67 & $y=0.2 x+30$ & $y=0.2 x+26$ \\
\hline$S M E_{\text {aver }}$ and $E_{\text {sw aver }}$ & 0.77 & 0.81 & $y=146 x+328$ & $y=131 x+362$ \\
\hline$A E_{\text {aver }}$ and $E_{\text {sw aver }}$ & 0.67 & 0.66 & $y=97 x+367$ & $y=95 x+353$ \\
\hline$K_{\text {paver }}$ and $E_{\text {sw aver }}$ & 0.66 & 0.77 & $y=5 x+34$ & $y=6 x+24$ \\
\hline$S M E_{\text {aver }}$ and $\left|B_{z \text { aver }}\right|$ & 0.62 & 0.73 & $y=45 x+447$ & $y=50 x+387$ \\
\hline$A E_{\text {aver }}$ and $\left|B_{z \text { aver }}\right|$ & 0.57 & 0.59 & $y=32 x+433$ & $y=37 x+370$ \\
\hline$K_{\text {paver }}$ and $\left|B_{z \text { aver }}\right|$ & 0.44 & 0.53 & $y=1.3 x+40$ & $y=1.9 x+30$ \\
\hline
\end{tabular}

types $S M E_{\text {aver }}$ increases linearly with $E_{\text {swaver }}$ and $\mid B_{z \text { aver }}$ ffeoc noitalerroc esolc dna hgih era erehT .|icients between $S M E_{\text {aver }}$ and $E_{\text {swaver }}$ for CIR $(r=0.77)$ and ICME $(r=0.81)$ events, whereas the correlation coefficients between $S M E_{\text {aver }}$ and $\left|B_{\text {zaver }}\right|$ for CIR $(r=0.62)$ and ICME $(r=0.73)$ are lower and markedly different (Figure 2, $b, d$ ).

\section{DISCUSSION AND CONCLUSIONS}

In this paper, we have examined the geomagnetic index of auroral activity $S M E$, which, unlike $A E$ and $K_{\mathrm{p}}$, takes into account the equatorward drift of ionospheric currents and allows for a more accurate assessment of the intensity of ionospheric currents during magnetic storms. As magnetic storm parameters, we took the rate of magnetic storm development and $D s t_{\min }$. Figure 1, $a$, $c$ shows that the SME index correlates with the magnetic storm development rate for CIR $(r=0.5)$ and ICME $(r=0.54)$ events. There is however a difference in angular coefficients in the regression equations between $S M E_{\text {aver }}$ and $|\Delta D s t| / \Delta T$ (see Table). Unlike $S M E$, the correlation coefficients between $|\Delta D s t| / \Delta T$ and $A E$ and $K_{\mathrm{p}}$ differ greatly for CIR and ICME events. For $A E$, the correlation coefficient for ICME $(r=0.29)$ is significantly lower than that for CIR $(r=0.51)$, whereas for $K_{\mathrm{p}}$, on the contrary, the correlation coefficient for ICME is slightly higher than that for CIR (see Table).

The magnetic storm velocity, or rather its variation $\Delta D s t / \Delta T$, depends on interplanetary medium parameters. A set of coupling functions for different combinations of SW/IMF parameters, including those determining $D s t$-index variations, has been proposed (e.g., [Burton et al., 1975; Newell et al., 2007; Borovsky, Birn, 2014; Dremuhina et al., 2018b]). Analysis has shown [Burton et al., 1975; Maltsev, Rezhenov, 2003] that there is a strong dependence of $\Delta D s t / \Delta T$ on southward IMF $B_{z}$. It is in the southward IMF $B_{z}$ component that the SW type manifests itself. For example, in ICME (MC and ejecta), the magnetic field has a structure in the form of a rope, and inside it the magnetic pressure prevails over the thermal one $\beta<1$. The magnetic cloud, unlike ejecta, has a higher ( $>10 \mathrm{nT})$ and more regular magnetic field. In CIR, plasma has an increased density and temperature, and the thermal pressure prevails over the magnetic pressure $\beta>1$. Figure $2, b, d$ shows that some of CIR-induced magnetic storms have small values of southward IMF $B_{z}$. This is probably why for CIR the angular coefficient of the regression equation between $S M E_{\text {aver }}$ and $|\Delta D s t| / \Delta T$ is lower than for ICME.

Khorosheva [2007] has reported findings of studies of the relationship between the shift of magnetospheric boundaries and the magnetic storm strength. According to this study, the Dst index makes it possible to estimate the position of boundaries of aurora and electrojets during magnetic storms. $D s t_{\min }$ determines the minimum latitude of the occurrence of auroras and electrojets during magnetic storms. The latitude of auroral electrojets during CIR and ICME events does not affect the $S M E$ index, as opposed to the $A E$ and $K_{\mathrm{p}}$ indices. As seen in Figure $1, b$, $d$, there are high and close correlation coefficients between $S M E$ and $D s t_{\min }$ during CIR $(r=0.67)$ and ICME $(r=0.6)$ events. There are no differences in the regression equations for CIR and ICME events either. The SW type has no effect on the relationship between $S M E_{\text {aver }}$ and $\left|D s t_{\text {min }}\right|$.

In this work, as in [Boroyev et al, 2020], to analyze the SW effect on auroral disturbances during magnetic storms, we have examined the SW electric field and IMF $B_{z}$. We have obtained higher and closer correlation coefficients between $S M E_{\text {aver }}$ and $E_{\text {swaver }}$ (see Table) as compared to $A E$ and $K_{\mathrm{p}}$. There are no noticeable differences in the angular coefficients in the regression equations between $S M E_{\text {aver }}$ and $E_{\text {swaver, }}$ and no relationship with the SW type (CIR/ICME) has been found either. The dependence of $S M E$ on $E_{\mathrm{sw}}$ is more pronounced than on $A E$. For $\mathrm{SW}$ of both types, a closer relationship is observed between $S M E_{\text {aver }}$ and $\left|B_{\text {zaver }}\right|$, yet the correlation coefficient for ICME events $(r=0.73)$ is higher than for CIR events $(r=0.62)$. Despite the difference in the correlation coefficients between $S M E_{\text {aver }}$ and $\left|B_{z \text { aver }}\right|$, the angular coefficients in 
the regression equations between $S M E_{\text {aver }}$ and $\left|B_{z \text { aver }}\right|$ for SW of both types have similar values (see Table). Comparative analysis of the correlation coefficients between $S M E_{\text {aver }}, A E, K_{\mathrm{p}}$ and the magnetic storm and interplanetary medium parameters, as well as the respective regression equations, allows us to conclude that $K_{\mathrm{p}}$, unlike $A E$, more accurately describes auroral activity in magnetic storm main phases during CIR and ICME events.

SW of each type has a certain set of parameters (see, e.g., [Yermolaev et al., 2010]). One of the important SW parameters is the southward IMF $B_{z}$ component whose geoeffectiveness is associated with the SW electric field. It is at the southward $B_{\mathrm{z}}$ component that most magnetospheric-ionospheric disturbances are recorded, and the difference between SW types is manifested in its value. For example, Figure 2 indicates that during CIR events most magnetic storms have low values of the southward $B_{z}$ component. Variations in the geomagnetic indices during the magnetic storm main phase depend on SW type. It has been shown [Plotnikov, Barkova, 2007; Yermolaev et al., 2010; Nikolaeva et al., 2011; Dremukhina et al., 2018a] that during magnetic storm main phases the dependences of $A E, a p, K_{\mathrm{p}}$ on $E_{\mathrm{sw}}$ are nonlinear for MC (possibly for all ICME events) and linear for CIR events. The nonlinear character is observed at high values of the SW electric field $\left(E_{\mathrm{sw}}>11\right.$ $\mathrm{mV} / \mathrm{m}$ ). According to [Plotnikov, Barkova, 2007; Yermolaev et al., 2017; Nikolaeva et al., 2017; Dremukhina et al., 2018a], the difference between variations in the indices for SW of different types may be due to the fact that along with southward IMF $B_{z}$ it is also necessary to take into account other SW parameters (for example, SW pressure, IMF turbulence) whose values differ for SW of different types.

Unlike the dynamics of the $A E$ and $K_{\mathrm{p}}$ indices, the dynamics of $S M E$ in magnetic storm main phases during CIR and ICME events does not depend on SW type. We have obtained high correlation coefficients between $S M E$ and the SW electric field (southward IMF $B_{z}$ ); and the regression equations between $S M E$ and $E_{\mathrm{sw}}$ (IMF $B_{z}$ ) for CIR and ICME events, taking into account the error in calculating the coefficients in the regression equations (within the error of $\pm 10 \%$ ), differ slightly. It should however be noted that in this work we have selected magnetic storms with $\left|D s t_{\mathrm{min}}\right|<130 \mathrm{nT}$. In [Plotnikov, Barkova, 2007; Yermolaev et al., 2010, 2017; Nikolaeva et al., 2017; Dremukhina et al., 2018a], stronger magnetic storms $\left|D s t_{\min }\right|>130 \mathrm{nT}$ have also been analyzed. It is possible that in magnetic storms with $\left|D s t_{\min }\right|<130$ nT during CIR and ICME events, the dynamics of the geomagnetic indices is largely determined by southward IMF $B_{z}$. Other SW parameters have little effect on the geomagnetic indices.

The results of the analysis lead to the following conclusions:

1. In magnetic storm main phases during CIR and ICME events, a stronger correlation is shown to be between $S M E_{\text {aver }}$ and the interplanetary medium parameters, as compared to $A E$ and $K_{\mathrm{p}}$. We have obtained close correlation coefficients between $S M E_{\text {aver }}$ and the SW electric field (southward IMF $B_{z}$ ) for CIR and ICME events.

2. We have found that $S M E_{\text {aver }}$ correlates with the rate of magnetic storm development and $\left|D s t_{\min }\right|$ for CIR and ICME events, while no relationship with SW type (CIR/ICME) is observed.

3. We have established that $K_{\mathrm{p}}$, unlike $A E$, more accurately describes the dynamics of auroral currents in magnetic storm main phases during CIR and ICME events.

The work was carried out in the framework of Government Assignment (State Registration Number AAAA A21-121012000007-4).

\section{REFERENCES}

Akasofu S.-I., Chapman S. Solnechno-zemnaya fizika. Chast'1. [Solar-Terrestrial Physics. Pt. 1]. Moscow, Mir Publ., 1974, 384 p. (In Russian). (English edition: Akasofu S.-I., Chapman S. Solar-Terrestrial Physics. Oxford, Clarendon Press, 1972, 901 p.)

Bartels J. The standardized index $K_{\mathrm{s}}$ and the planetary index $K_{\mathrm{p}}$. IATME Bull. 1949, no. 12b, pp. 97-120.

Borovsky J.E., Denton M.H. Differences between CME driven storms and CIR driven storms. J. Geophys. Res. 2006, vol. 111, A07S08. DOI: 10.1029/2005JA011447.

Borovsky J.E., Birn J. The solar wind electric field does not control the dayside reconnection rate. J. Geophys. Res. 2014, vol. 119, pp. 751-760. DOI: 10.1002/2013JA019193.

Boroyev R.N., Vasiliev M.S. Substorm activity during the main phase of magnetic storms induced by the CIR and ICME events. Adv. Space Res. 2018, vol. 61, pp. 348-354.DOI: 10.1016/j.asr.2017.10.031.

Boroyev R.N., Vasiliev M.S., Baishev D.G. The relationship between geomagnetic indices and the interplanetary medium parameters in magnetic storm main phases during CIR and ICME events. J. Atmos. Solar-Terr. Phys. 2020, vol. 204, 105290. DOI: 10.1016/j.jastp.2020.105290.

Burton R.K., McPherron R.L., Russell C.T. An empirical relationship between interplanetary conditions and Dst. J. Geophys. Res. 1975, vol. 80, pp. 4204-4214. DOI: 10.1029/JA 080i031p04204.

Cramer W.D., Turner N.E., Fok M.C., Buzulukova N.Y. Effects of different geomagnetic storm drivers on the ring current: CRCM results. J. Geophys. Res. 2013, vol. 118, pp. 1062-1073. DOI: $10.1002 /$ jgra.50138.

Davis T.N., Sugiura M. Auroral electrojet activity index $A E$ and its universal time variations. J. Geophys. Res. 1966, vol. 71, pp. 785-801. DOI: 10.1029/JZ071i003p00785.

Dremukhina L.A., Lodkina I.G., Yermolaev Y.I. Relationship between the parameters of various solar wind types and geomagnetic activity indices. Cosmic Res. 2018a, vol. 56, no. 6, pp. 426-433. DOI: 10.1134/S0010952518060011.

Dremukhina L.A., Lodkina I.G., Yermolaev Y.I. Statistical study of the effect of different solar wind types on magnetic storm generation during 1995-2016. Geomagnetism and Aeronomy. 2018b, vol. 58, no. 6, pp. 737-743. DOI: 10.1134/S0016793218060038.

Du A.M., Wang K.T., Luo H., Tsurutani B.T., JesperGjerloev, Wei Sun, Wang Y., Jiaming Ou, Yasong Ge. Coupling of semiannual and annual variations in the SuperMAG SML and SMU indices. Planet. Space Sci. 2018, vol. 158, pp. 8795. DOI: 10.1016/j.pss.2018.05.001.

Gonzalez W.D., Joselyn J.A., Kamide Y., Kroehl H.W., Rostoker G., Tsurutani B.T., Vasyliunas V.M. What is a geomagnetic storm? J. Geophys. Res. 1994, vol. 99, iss. A4, pp. 5771-5792. DOI: 10.1029/93JA02867. 
Gonzalez W.D., Tsurutani B.T., Gonzalez A.L.C. Interplanetary origin of geomagnetic storms. Space Sci. Rev. 1999, vol. 88, pp. 529-562. DOI: 10.1023/A:1005160129098.

Grafe A., Feldstein Y.I. About the relationship between auroral electrojets and ring currents. Ann. Geophys. 2000, vol. 18, pp. 874-886. DOI: 10.1007/s00585-000-0874-4.

Grafe A., Bespalov P.A., Trakhtengerts V.Y., Demekhov A.G. Afternoon mid-latitude current system and low-latitude geomagnetic field asymmetry during geomagnetic storms. Ann. Geophys. 1997, vol. 15, pp. 1537-1547. DOI: 10.1007/s00585-997-1537-5.

Guo J., Feng X., Emery B.A., Zhang J., Xiang C., Shen F., Song W. Energy transfer during intense geomagnetic storms driven by interplanetary coronal mass ejections and their sheath regions. J. Geophys. Res. 2011, vol. 116, A05106. DOI: 10.1029/ 2011jA016490.

Kane R.P. How good is the relationship of solar and interplanetary plasma parameters with geomagnetic storms? $J$. Geophys. Res. 2005, vol. 110, A02213. DOI: 10.1029/2004JA 010799 .

Kane R.P. Scatter in the plots of $D s t(\min )$ versus $B_{\mathrm{Z}}(\mathrm{min})$. Planet. Space Sci. 2010, vol. 58, pp. 1792-1801. DOI: 10.1016/j.pss.2010.07.026.

Khorosheva O.V. Relation of geomagnetic disturbances to the dynamics of the magnetosphere and the parameters of the interplanetary medium. Geomagnetism and Aeronomy. 2007, vol. 47 , no. 5, pp. 543-547. DOI: 10.1134/S0016793207050015.

Maltsev Yu.P., Rezhenov B.V. Relation of the Dst index to solar wind parameters. International Journal of Geomag netism and Aeronomy. 2003, vol. 4, no. 1, pp. 1-9.

Newell P.T., Gjerloev J.W. Substorm and magnetosphere characteristic scales inferred from the SuperMAG auroral electrojet indices. J. Geophys. Res. 2011, vol. 116, A12232. DOI: $10.1029 / 2011$ JA016936.

Newell P.T., Gjerloev J.W. SuperMAG-based partial ring current indices. J. Geophys. Res. 2012, vol. 117, A05215. DOI: $10.1029 / 2012 J A 017586$

Newell P.T., Sotirelis T., Liou K., Meng C.-I., Rich F.J. A nearly universal solar wind-magnetosphere coupling function inferred from 10 magnetospheric state variables. $J$. Geophys. Res. 2007, vol. 112, A01206. DOI: 10.1029/ 2006JA012015.

Newell P.T., Gjerloev J.W., Mitchell E.J. Space climate implications from substorm frequency. J. Geophys. Res. 2013 , vol. 118, pp. 6254-6265. DOI: 10.1002/jgra.50597.

Nikolaeva N.S., Yermolaev Y.I., Lodkina I.G. Dependence of geomagnetic activity during magnetic storms on the solar wind parameters for different types of streams. Geomag netism and Aeronomy. 2011, vol. 51, no. 1, pp. 49-65. DOI: 10.1134/S0016793211010099.

Nikolaeva N.S., Yermolaev Y.I., Lodkina I.G., Yermolaev M.Y. Does magnetic storm generation depend on the solar wind type? Geomagnetism and Aeronomy. 2017, vol. 57, no. 5, pp. 512-518. DOI: 10.1134/S0016793217050152.

Plotnikov I.Ya., Barkova E.S. Advances in space research nonlinear dependence of $D s t$ and $A E$ indices on the electric field of magnetic clouds. Adv. Space Res. 2007, vol. 40, pp. 1858-1862. DOI: 10.1016/j.asr.2007.09.025.

Sugiura M. Hourly values of the equatorial Dst for IGY. Annales of the International Geophysical Year. 1964, vol. 35. pp. 9-45.

Yermolaev Yu.I., Nikolaeva N.S., Lodkina I.G., Yermolaev M.Yu. Catalog of large-scale solar wind phenomena during 1976-2000. Cosmic Res. 2009, vol. 47, no. 2, pp. 81-94. DOI: $10.1134 / \mathrm{S} 0010952509020014$

Yermolaev Yu.I., Nikolaeva N.S., Lodkina I.G., Yermolaev M.Yu. Specific interplanetary conditions for CIR-, Sheath-, and ICME-induced geomagnetic storms obtained by double superposed epoch analysis. Ann. Geophys. 2010, vol. 28, pp. 2177-2186. DOI: 10.5194/angeo-28-1-2010.

Yermolaev Y.I., Nikolaeva N.S., Lodkina I.G., Yermolaev M.Y. Geoeffectiveness and efficiency of CIR, sheath, and ICME in generation of magnetic storms. J. Geophys. Res. 2012, vol. 117, A00L07. DOI: 10.1029/2011JA017139.

Yermolaev Y.I., Lodkina I.G., Nikolaeva N.S., Yermolaev M.Y. Does the duration of the magnetic storm recovery phase depend on the storm development rate in its main phase? Geomagnetism and Aeronomy. 2015, vol. 55, no. 4, pp. 421-424. DOI: 10.1134/S0016793215040039.

Yermolaev Y.I., Lodkina I.G., Nikolaeva N.S., Yermolaev M.Y. Dynamics of large-scale solar-wind streams obtained by the double superposed epoch analysis: 2. Comparisons of CIRs vs. Sheaths and MCs vs. Ejecta. Solar Phys. 2017, vol. 292, 193. DOI: 10.1007/s11207-017-1205-1.

Yermolaev Y.I., Lodkina I.G., Dremukhina L.A., Yermolaev M.Y., Khokhlachev A.A. What solar-terrestrial link researchers should know about interplanetary drivers. Universe. 2021, vol. 7, iss. 5, 138. DOI: 10.3390/universe7050138.

URL: https://supermag.jhuapl.edu (accessed March 2, 2021).

URL: http://wdc.kugi.kyoto-u.ac.jp/dstae/index.html (accessed March 2, 2021) 2021).

URL: ftp.iki.rssi.ru/pub/omni/catalog (accessed March 2,

URL: http://www.omniweb.com (accessed March 2, 2021).

How to cite this article:

Boroyev R.N., Vasiliev M.S. Study of auroral activity according to the SME index at the main phase of magnetic storms during CIR and ICME events. Solar-Terrestrial Physics. 2021. Vol. 7. Iss. 4. P. $18-23$. DOI: $10.12737 /$ stp-74202103 